\title{
Family Support and Stigma among Patients Attending a Tertiary Care Hospital in Bangladesh
}

\author{
Jahan Na , Maruf MMº, Sultana Bc, Habib SH ${ }^{\mathrm{d}}$, Khan MZR ${ }^{\mathrm{e}}$, Begum A ${ }^{\mathrm{f}}$
}

\begin{abstract}
Background: Mental illness stigma continues to be a major barrier for individuals with mental illness.

Methods: This study was a descriptive cross-sectional study conducted among the mentally ill patients attending Out Patient Department (OPD) in a tertiary care hospital of Dhaka city of Bangladesh, during the period from May 2013 to November 2013. The objective of the study was to assess the family support and stigma among the respondents. A total of 151 patients of 18 to 60 years attending in the OPD, National Institute of Mental Health (NIMH), Dhaka were selected purposefully to conduct the study. An informed consent was taken from the patients or care givers. Information about socio-demographic and psychosocial data was collected using the questionnaire designed by the researcher based on Factors Influencing Neuroleptic Medication Taking Scale (FNIMTS). Diagnoses of mental illness were done according to Diagnostic and Statistical Manual of Mental Disorders Text Revision (DSM-IV-TR) by psychiatrists.
\end{abstract}

Results: The commonest diagnoses among the respondents were the schizophrenias (56.3\%), bipolar mood disorders (23.8\%) and substance related disorder (6.6\%). Most were insightful into their mental illness (89.4\%). Most of the respondents rated their perception of family support as good (66.2\%). Most of the respondents believed that they would be treated differently if people knew they had a mental illness (57.6\%) or on drugs for mental illness (54.3\%). Among the presently employed respondents (21.85\%) most (57.75\%) were uncomfortable with the idea of telling employer about mental illness or being on drugs for it.

Conclusion: A bridge for communication between people with mental illness, their families, and health care practitioners, may be a useful framework for guiding efforts to reduce stigma. Community-based participatory research principles and lived experiences are crucial elements in stigma reduction endeavors.

(BIRDEM Med J 2017; 7(2): 148-154)

Author Information

a. Dr. Nasim Jahan, Assistant Professor, BIRDEM General Hospital and Ibrahim Medical College, Dhaka.

b. Dr. Mohammad Muntasir Maruf, Assistant Professor, Department of Psychiatry, Shaheed M. Monsur Ali Medical College, Sirajganj.

c. Dr. Bushra Sultana, HMO, National Institute of Mental Health (NIMH), Dhaka.

d. Samira Humaira Habib, Principal Research Officer, HEU, BADAS, Dhaka.

e. Dr. Muhammad Zillur Rahman Khan, Assistant Professor, Department of Child, Adolescent and Family Psychiatry, NIMH, Dhaka.

f. Prof. Anwara Begum, Professor and Head of the Department of Psychiatry, BIRDEM General Hospital and Ibrahim Medical College, Dhaka.

Address of correspondence: Dr. Nasim Jahan, Assistant Professor, BIRDEM General Hospital and Ibrahim Medical College, Dhaka, email: njahan.bird@gmail.com.

Received: July 25, 2016

Accepted: 28 February 2017

\section{Introduction}

In Southeast Asian cultures, the family is an individual's religious, economic, political, and social core. It is the first loyalty and primary obligation. The family obligation means that members must care for each other. For example, grandparents and older siblings care for the young and adult children care for their aging parents. Family relations and functions are clearly and elaborately defined. Mutual two way obligations connect families, helping them with tasks such as parenting and creating strong bonds.

Traditionally, Southeast Asians tend to have a large extended family, usually up to three or four generations living together. Nuclear families typically have four to eight children, depending on socioeconomic status and ethnic group. 
Southeast Asian children are taught early in life about the importance of relationships and obligations within the family and society. As children mature, they develop a moral obligation and primary loyalty to the family. Family usually takes good care of the sick family members in our country.

The traditional family is structured by age and gender. Males are usually dominant and authoritarian. Women in traditional Southeast Asian cultures are considered subordinate. Traditionally, girls and boys have different roles and are treated differently. Males get priority in different aspects including health care facilities. Mental health and possible care of it have not become a high priority in most Asian countries, despite the recent emphasis at international level. ${ }^{1}$

Most people in the general population have a mixture of little and wrong information about mental illnesses, alongside wary and cautious attitudes about mentally ill people. It is therefore understandable that they are often slow to recognize developing mental health problems in themselves, in family members or in friends, and are reluctant to seek help for fear of the consequences. Recently we have begun to understand the power of these barriers to finding treatment and care of mental health. For example studies from several countries have consistently found that even after a family member has developed clear-cut signs of a psychotic disorder, on average it is over a year until the unwell person first receives assessment and treatment. $^{2}$

Stigma impacts care seeking at personal, provider, and system levels. The relationship between stigma and care seeking may be moderated by three additional variables, knowledge, culture, and network. Researchers have argued that improper or insufficient knowledge about mental illness and its treatment leads to stigmatizing attitudes and discriminatory behavior. In turn, knowledge and attitudes have been shown to be predictors of care seeking and participation. Burden may be exacerbated by family or "courtesy" stigma, a term coined by Goffman that reflects families, friends, and others being objects of prejudice and discrimination because of their association with the person with mental illness. Like people with mental illness, some of their family members may be harmed by public and by selfstigma. $^{3}$

Despite many problems facing psychiatry and mental health care in Asia, there are several strengths that cannot be ignored and indeed are worth preserving, if possible. Among these is the still largely intact family cohesion that is a resource for support of the mentally ill. Most mental patients in Malaysia are visited when admitted in hospitals on a daily basis and taken back to their own homes when discharged. Rejection of the mentally ill is still fairly uncommon and occurs in conditions of abject poverty and economic privation. This is however may change rapidly with provision of insurance cover or reimbursement systems that are designed to encourage long hospital stays, as in Japan and Korea, where the average length of stay in private hospitals may exceed a year.

Traditional belief systems encourage care of the disabled and mentally ill in many cultures in Asia. Thus many mentally ill are said to be possessed by spirits and capable of special powers and sometimes even revered. While this may deny them access to treatment, their social status is elevated beyond the stigmatization that may otherwise occur. Religious practices and belief in religion are remarkably strong in most of Asia and this is another source of both caring and treatment for the mentally ill. Many treatment centers focus on prayers for the cure or wellbeing of the ill person and this appears to be beneficial to some. ${ }^{4}$

There is ongoing need for anti-stigma campaigns to reduce the fear \& misunderstandings about psychiatry. The professionals involved in mental health issues together with patients, families \& support organizations will need to work closely with the media for the change towards positive. Researches are needed in this field to explore the areas of attention to reduce stigma of mental illness in a developing country like Bangladesh. The objective of this study was to assess the family support and stigma among the respondents.

\section{Methods}

This was a descriptive cross-sectional study conducted among the patients attending the outpatient 
department of NIMH, Dhaka. The duration of study was seven months, starting from May 2013 to November 2013. In the study design, purposive sampling technique was used and a sample size of 151 was taken which is a valid sample size for this type of sampling technique to conduct a scientifically sound study.

A total 151 respondents of 18 to 60 years were selected purposefully according to inclusion criteria and informed written consent was taken from each patient and his or her attendant. During data collection, a semistructured questionnaire designed by the researcher based on Factors Influencing Neuroleptic Medication Taking Scale (FNIMTS) containing sociodemographic and other variables was used. ${ }^{7}, 17$ Diagnoses were done previously according to Diagnostic and Statistical Manual of Mental Disorders Text Revision (DSM-IV-TR) by psychiatrists. Selfreport was verified by the attendants as needed. Data were analyzed using Statistical Package for Social Sciences (SPSS), version 15.0 for Windows. Ethical clearance was taken from the ethical clearance committee of NIMH.

\section{Results}

The objective of the study was to find out the pattern of family support and stigma among the patients attending a tertiary care hospital for mental illness situated in Dhaka, Bangladesh. A total of 151 adults of 18 to 60 years attending in the OPD, NIMH Dhaka were included in the study.

\section{Socio demographic profile}

The study identified the socio demographic characteristics of the respondents including age, sex, religion, habitat, educational level, marital status and working status. It also included other relevant information about the family such as, type of family, number of family members and monthly expenditure of family.

Details of socio-demographic characteristics are illustrated in Table $1 \&$ Table 2 and Figure $1 \&$ Figure 2.
Table I. Socio-demographic characteristics of the study population $(\mathrm{n}=151)$

\begin{tabular}{|c|c|c|}
\hline Variable & Frequency & Percentage $(\%)$ \\
\hline \multicolumn{3}{|l|}{ Age } \\
\hline $18-23$ & 33 & 21.9 \\
\hline $24-29$ & 27 & 17.9 \\
\hline $30-35$ & 36 & 23.8 \\
\hline $36-41$ & 27 & 17.9 \\
\hline $42-47$ & 17 & 11.3 \\
\hline $48-60$ & 11 & 7.3 \\
\hline \multicolumn{3}{|l|}{ Sex } \\
\hline Male & 100 & 66.2 \\
\hline Female & 51 & 33.8 \\
\hline \multicolumn{3}{|l|}{ Religion } \\
\hline Islam & 145 & 96.0 \\
\hline Hinduism & 5 & 3.3 \\
\hline Christianity & 1 & 0.7 \\
\hline \multicolumn{3}{|l|}{ Residence } \\
\hline Urban & 85 & 56.3 \\
\hline Semi-urban & 20 & 13.2 \\
\hline Rural & 39 & 25.8 \\
\hline Slum & 7 & 4.6 \\
\hline \multicolumn{3}{|c|}{ Marital status } \\
\hline Single & 62 & 41.1 \\
\hline Married & 70 & 46.4 \\
\hline Widower & 2 & 1.3 \\
\hline \multicolumn{3}{|l|}{ Separated } \\
\hline Divorced & 413 & 2.68 .6 \\
\hline
\end{tabular}

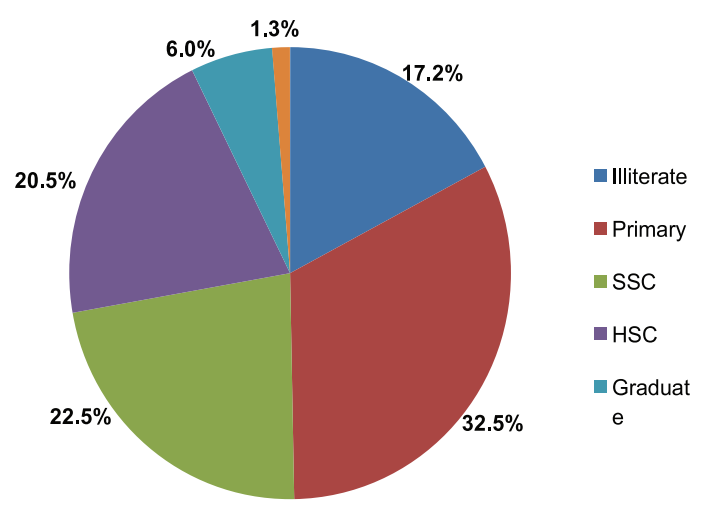

Figure 1. Level of education received by the respondents $(n=151)$ 
Table II. Characteristics of the family of study population $(\mathrm{n}=151)$

\begin{tabular}{lll} 
Variable & Frequency & Percentage (\%) \\
\hline
\end{tabular}

Type of family

Nuclear 96

Non-nuclear

51

33.8

Others

4

Number of family members

$\begin{array}{ccc}1-2 & 7 & 4.6 \\ 3-4 & 53 & 35.1 \\ 5-6 & 57 & 37.7 \\ 7-8 & 27 & 17.9 \\ \geq 9 & 7 & 4.6\end{array}$

Family expenditure (in Taka)

\begin{tabular}{lcc}
$\leq 4000$ & 9 & 6.0 \\
$4001-7000$ & 30 & 19.9 \\
$7001-10000$ & 50 & 33.1 \\
$10001-13000$ & 24 & 15.9 \\
$13001-16000$ & 20 & 13.2 \\
$\geq 16001$ & 18 & 11.9 \\
\hline
\end{tabular}
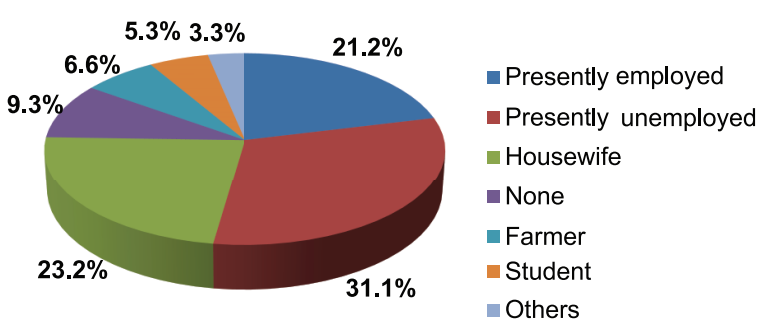

Figure 2. Present working status of the respondents $(n=151)$

\section{Clinical variables}

The commonest diagnoses among the respondents were the schizophrenias (56.3\%), bipolar mood disorders $(23.8 \%)$ and substance related disorder (6.6\%). Most were insightful into their mental illness (89.4\%).

Table 3 \& Fig 3 Illustrates the summery of illness related characteristics.
Table III. Clinical variables of the respondents $(\mathrm{n}=151)$

Variable

Frequency Percentage (\%)

\section{Diagnosis}

\begin{tabular}{lcc} 
Schizophrenia & 85 & 56.3 \\
BMD & 36 & 23.8 \\
SRD & 10 & 6.6 \\
MDD & 4 & 2.6 \\
OCD & 4 & 2.6 \\
DCD & 4 & 2.6 \\
Schizoaffective disorder & 3 & 2.0 \\
Others & 5 & 3.3 \\
\hline
\end{tabular}

BMD: Bipolar Mood Disorder OCD: Obsessive Compulsive Disorder, SRD: Substance Related Disorder DCD: Dissociative conversion Disorder MDD: Major Depressive Disorder

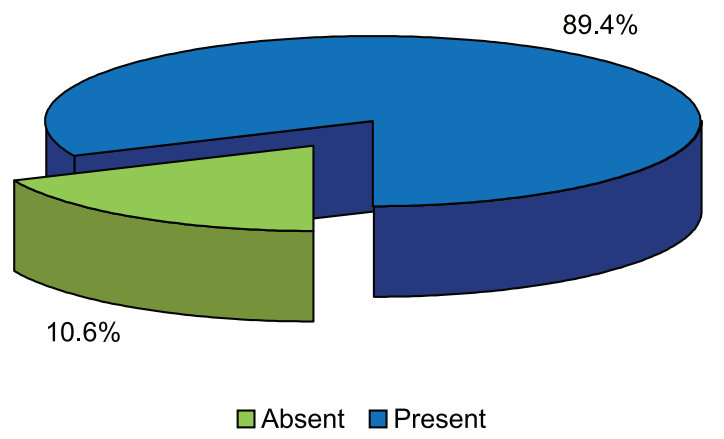

Figure 3. Proportion of Insight among the respondents $(n=151)$

\section{Psychosocial variables}

Perceived illness related believe of all the respondents and stigma among the respondents having insight was assessed by some questions as psychosocial variable.

Illustrations of summery statistics for the psychosocial variables are illustrated in table $4 \& 5$. 
Table IV. Perceived Illness related believes of the respondents $(\mathrm{n}=151)$

\begin{tabular}{lcc} 
Variable & Frequency & Percentage (\%) \\
\hline Perceived cause of & illness & Predominantly \\
Spiritual & 21 & 13.9 \\
Biological & 23 & 15.2 \\
Psychosocial & 54 & 35.8 \\
Not known & 37 & 24.5 \\
Not applicable & 16 & 10.6 \\
Perceived prognosis of illness & \\
Good & 110 & 72.8 \\
Fair & 23 & 15.2 \\
Poor & 2 & 1.3 \\
Not applicable & 16 & 10.6 \\
Perceived level of family support & \\
Good & 100 & 66.2 \\
Fair & 42 & 27.8 \\
Poor & 9 & 6.0 \\
Respondents believe medications are needed? \\
Yes & 125 & 82.8 \\
No & 10 & 6.6 \\
Not applicable & 16 & 10.6 \\
Respondents believe hospital visits are needed? \\
Yes & 129 & 85.4 \\
No & 6 & 4.0 \\
Not applicable & 16 & 10.6 \\
\hline
\end{tabular}

\section{Discussion}

The study included highest number $(23.8 \%)$ of patients from the age range of 30 to 35 years followed by $(21.9 \%)$ from the range of 18-23 years with the mean age of 32.77 years. This trend of age does not differ much from the result of predominant 20 to 29 years $(48.5 \%)$ age group the second highest group that is $30-39$ years $(29.7 \%)$ described in a OPD based earlier study conducted in NIMH. ${ }^{5}$ The over-presentation of this particular age group in this study does not seem to be a selection bias as mental health of elderly patients is still neglected in our country.

In this study majority of the respondents were male $(66.2 \%)$. This finding is consistent with the previous study of our country where male respondents were
Table V. Stigma of the respondents (having insight) about the illness $(n=135)$

Variable $\quad$ Frequency $\quad$ Percentage $(\%)$

Stigma of others if knows about mental illness

$\begin{array}{lll}\text { Yes } & 81 & 60.0 \\ \text { No } & 54 & 40.0\end{array}$

Stigma of others if knows on medication for mental illness

$\begin{array}{lll}\text { Yes } & 76 & 56.3 \\ \text { No } & 59 & 43.7\end{array}$

Fear of others put off taking medication

$\begin{array}{ccc}\text { Yes } & 8 & 5.9 \\ \text { No } & 127 & 94.1\end{array}$

Can tell employer about mental illness

$\begin{array}{ccc}\text { Yes } & 14 & 10.4 \\ \text { No } & 17 & 12.6 \\ \text { Not applicable } & 104 & 77.0\end{array}$

Can tell employer about taking medication for mental illness

\begin{tabular}{lcc} 
Yes & 14 & 10.4 \\
No & 17 & 12.6 \\
Not applicable & 104 & 77.0 \\
\hline
\end{tabular}

majority (67.7\%) but in Pakistan, male female ratio of respondents in a study on Non-adherence in Karachi was found to be almost equal. ${ }^{5,6}$ Not only that, in Nigeria female respondents were found to be a bit higher $(51.8 \%){ }^{7}$ Most of the study results of Bangladesh is consistent with male domination of psychiatric illness in children. ${ }^{8,9,10}$ This gender inequity may be explained by the fact that more attention and care is given to male children and any deviation of their behavior and scholastic performance is more likely to be noted and reported by parents. Thus female children get less opportunity to seek health care which is unfortunately enrooted in our culture. This picture continues even in the adult life which can be concluded from this study. 
Educational status of this study population represented highest $(32.5 \%)$ from the primary level. Thus the maximum (49.7\%) were in the group of illiterate to primary level. In another way this result is very much consistent as a significant portion (43.7\%) of respondents of this study population were not from urban, which is educationally advantaged.

The result of the dwelling status reflected the urban predominance $(56.3 \%)$ among the respondents. It is clearly explainable by the link of study place which is in the core of Dhaka city. Urbanization contributed by better availability of health facilities and greater awareness of people explains this correlation.

The result showed $96.0 \%$ cases with the religion of Islam and $4.0 \%$ in other religion. The finding is somehow usual in comparison with the Muslim/non-Muslim distribution of the population of our country which is $90 \%$ and $10 \%$ respectively, reported in population census, 2001.

Almost half of the respondents were in married group $(46.4 \%)$ but the rate has dropped a bit than the previous study $(51.7 \%){ }^{5}$ which may be due to difference in study population.

Regarding occupation, $21.2 \%$ was found to be employed at the time of interview, $49.7 \%$ in the previous study. ${ }^{5}$ This difference may be due to the fact that we had another group who were employed but not now due to illness $(31.1 \%)$ temporarily or permanently. Thus the total of employment together would be $52.3 \%$ indicating that mentally ill persons are now having more quality of life than before. In Pakistan the employment of this group was found $28.90 \%$. In Nigeria employed was $44.5 \%$ and in two centers of Jamaica $28.5 \%$ and $29.0 \%$ among the respondents. ${ }^{7,11}$ Except Nigeria the findings are nearer to our finding. Nigerian study may differ as they had no extra category labeled as "others". In our study, housewives were $23.2 \%$ but in previous one $15.9 \%$, may indicate increased caring for the female members of the family now-a-days. Student was 5.3\%, comparatively lower than previous (13.1\%) as that study took age range from 10 and above. ${ }^{5}$

Family characteristics were not included in previous studies, but living circumstances in some studies. We found only $4(2.6 \%)$ respondents living in places other than family. Among rest of them majority were living in nuclear family $(63.6 \%)$. Even when nuclear family predominated members per family was found to be 5-6
$(37.7 \%)$ which seems a bit higher. In the view of socio economic condition, $33.1 \%$ was found having family expenditure in the range of 7001-10000 taka. Only 6.0\% in the range up to 4000 and $11.9 \%$ in the range above 16,001 . It may indicate that rest of these lower middle $\&$ middle class families prefer to seek help from the government hospital more than the affluent society.

Clinical variable showed that Schizophrenia was the diagnosis of over half of the respondents $(56.3 \%)$ followed by BMD (23.8\%) which is similar to previous local study. Interesting finding is that SRD was increased tremendously from $0.7 \%$ of the previous to $6.6 \%$ in this study over last 10 years. ${ }^{5}$

Insight was present in the majority participant (89.4\%) who was assessed by the question whether the participant treated for mental illness or not. Their views of need to continue hospital visit and current medication was positive (yes for $95.5 \%$ and $92.6 \%$ respectively).

In this study, participant assessed family support to be good in the maximum cases and said that relative encourage to take medicine and attend clinic (89.4\% and $90.1 \%$ respectively). The role played by the family in the care of mentally ill is substantial. A previous study reported that post discharged, patient who benefited from relative support were more likely to comply with clinic appointment and adherence to treatment. ${ }^{12}$

Stigma has been consistently reported in the literature to adversely affect health seeking behavior and treatment outcome of mental illness.$^{5}$ In a local study, the range of awareness about mental disorders was $85-15.13 \%$, range of acceptance of mentally ill patients was 71.42 $60.50 \%$, range of willingness to care for mentally ill was $63.54-15.13 \% .41 \%$ of the patients believed that black magic or evil spirits are the cause of mental illness. ${ }^{13}$ Another study showed that commonly believed reasons of mental illness were possessions of Zinnis, influence (upri) of pari, demons, evil spirits, kufri kalams, familial (stigma-attached) and low intelligence. For treatment most of the mentally ill persons (62.37\%) of our country first go to peer, fakir, huzur, kabiraj etc. ${ }^{14}$ A study conducted in 2009 found that $78 \%$ of psychiatric patients got treatment from traditional healers. ${ }^{15}$ In another study conducted in Chittagong Medical College, it was found that $80.65 \%$ of patients took one or more types of traditional treatment. ${ }^{16}$ 
Only the respondents who had insight were asked about cause and prognosis of their mental illness. Majority (72.8\%) expected good prognosis and thought the disease was due to the psychosocial origin (35.8\%). The percentage of believing it to be spiritual is being lowest (13.9\%).The finding is just opposite of that in a Nigerian study $^{5}$. This finding against our socio-cultural background may indicate those people who are more aware coming for treatment in hospital.

In this study, over half of respondent thought that they would be treated differently by people knowing they have mental illness or on drugs for mental illness. Not only were that over half of the respondent presently employed unwilling to tell their employers about mental illness but interesting to note that paradoxically only few $(7.9 \%)$ respondent said that fear of others put them of taking medication. This finding is similar to the previous studies. ${ }^{5,17}$

Although optimum care had been tried by the researcher in every steps of this study, still some limitations existed. The result should be interpreted in the light of the limitations like purposive sampling technique was followed in this study, so there could be some selection bias and the study was conducted in a selected hospital of Dhaka city so, the study population may not represent the whole population and limits the generalization of the result.

Mental health care is by no means standardized and extremely varied in Asia. Many so-called norms of psychiatric care in many economically highly developed countries may not only be not

applicable but highly detrimental to mental health care in many parts of Asia. Many aspects of mental health care are not available in vast areas of Asia and often substituted by a remarkably resilient, but not always highly successful, family care alternative and a strong heritage of traditional medical care for the mentally ill, that is very often the norm. ${ }^{4}$

Further large scale studies are required to explore the pattern of stigma in our country to deliver adequate mental health care to all.

Declaration: This paper was presented in World Psychiatry Association Regional Congress, Kochi, India, 2015.

\section{References}

1. Morrow R. Southeast Asian child rearing practices: Implications for child and youth care workers. Child and Youth Care Quarterly 1989; 18(4):273-87.
2. Thornicroft G, Rose D, Kassam A. Discrimination in health care against people with mental illness. International Review of Psychiatry 2007; 19(2): 113-22.

3. Corrigan PW, Druss BG, Perlick DA. The impact of mental illness stigma on seeking and participating in mental health care. Psychological Science in the Public Interest 2014; 15(2): 37-70.

4. Deva P. Mental health and mental health care in Asia. World Psychiatry 2002; 1(2): 118-20.

5. Rahman AHMM, Uddin N, Islam T, Azda C, Uddin J, Firoz AHM. Non-compliance among psychiatric patients- a hospital based study. Bang J Psychiatry 2003; 17(1):5-14.

6. Fawad T, Mansoor T, Zarmeneh A, Ameer AK, Asma T, Fahd $\mathrm{KS}$, et al. Factors associated with Non-adherence among Psychiatric patients at a Tertiary Care Hospital, Karachi, Pakistan: a questionnaire based cross sectional study. J Pak Med Assoc 2008; 58:432.

7. James BO, Omoaregba JO. Prevalence and predictors of poor medication adherence among out-patients at a psychiatric hospital in Benin city, Nigeria. International J Psychiatr Clin Practice 2011; 15:27-34.

8. Rabbani MG, Alam MF, Ahmed HU, Sarkar M, Islam MS, Anwar $\mathrm{N}$ et al. Prevalence of mental disorders, mental retardation, epilepsy and substance abuse in children. Bang $\mathrm{J}$ Psychiatry 2009; 23:11-52.

9. Rahim D, Ali SMS, Rabbani MG, Rahman MA. Analysis of psychiatric morbidity of outpatient children in Midford hospital, Dhaka. Bangladesh Med Res Counc Bull 1997; 23(2):60-62.

10. Sarkar M, Khan MJR, Rabbani MG, Alam MF, Firoz AHM, Alam MT et al. Pattern of psychiatric morbidity among the children and adolescents attending child guidance clinic of National Institute of Mental Health (NIMH), Dhaka. Bang J Psychiatry 2008; 22(1):11-21.

11. Andrea EP, Paul AB, Stan W, Janet L, Christopher ADC. Medication compliance among mentally Ill patients in public clinics in Kingston and St. Andrew, Jamaica. J Biomed Science Engineering 2010;3: 602-11.

12. Alex J. Why don't patients take their medicine? Reasons and solutions in psychiatry. Advances in psychiatric treatment 2007; 13:336-46.

13. Firoz AHM, Karim ME, Rahman AHMM, Zaman MM, Rabbani MG. Awareness, care and acceptance of mental health patients: A nationwide multicentric community survey in Bangladesh. Journal of NIMH 2009; 1(1): 9-30.

14. Rabbani MG, Islam MT. Awareness about mental illness and its treatment approach- A key information study. Bang J Psychiatry 2004; 18(1): 16-21.

15. Islam MT, Uddin MMJ, Ahmed HU, Rabbani MG. Treatment practices by traditional healers in treatment of psychiatric patients- A community centered study. Bang J Psychiatry 2009; 23(2): 30-35.

16. Alam MS, Haque SM. Traditional methods of treatment adopted in psychiatric illness. A CMH report. Bang J Psychiatry 1998; 12(2): 58-68.

17. Mccann TV, Boardman G, Clark E, LU S. Risk profiles for non-adherence to antipsychotic medications. Journal of Psychiatric and Mental Health Nursing 2008; 15: 622-29. 